\title{
Variabilidad de la síntesis de citoquinas por células dendríticas humanas estimuladas con los distintos serotipos de Aggregatibacter actinomycetecomitans
}

\author{
Variability in the cytokine synthesis by human dendritic \\ cells in response to different Aggregatibacter \\ actinomycetemcomitans serotypes
}

\author{
Díaz-Zúñiga $\mathrm{J}^{1}$, Melgar-Rodríguez $\mathrm{S}^{1}$ y Yáñez $\mathrm{JP}^{1}$, Álvarez $\mathrm{C}^{1}$, Rojas $\mathrm{C}^{1}$, Benítez $\mathrm{A}^{1}$, Ciuchi $\mathrm{P}^{1}$, Monasterio $\mathrm{G}^{1}$, Vernal $\mathrm{R}^{1}$
}

\begin{abstract}
RESUMEN
Objetivo: Sobre la base de la antigenicidad del polisacárido O del LPS, en A. actinomycetemcomitans se describen distintos serotipos bacterianos y entre ellos se ha especulado una patogenicidad e inmunogenicidad diferente. El objetivo de este trabajo es analizar las diferencias en la síntesis de citoquinas producidas por células dendríticas cuando son estimuladas con los distintos serotipos de $A$. actinomycetemcomitans. Metodología: Células dendríticas diferenciadas a partir de monocitos circulantes periféricos humanos fueron estimuladas a MOls $=10^{-1}-10^{-2}$ con los serotipos a, b y $\mathrm{c}$ de A. Actinomycetemcomitans. Mediante PCR y ELISA se evaluaron los niveles de expresión y secreción de citoquinas. Resultados: En las células dendríticas, la producción de citoquinas fue diferente ante los distintos serotipos de A. actinomycetemcomitans, con mayores niveles de secreción de IL-1 $\beta$, IL-6, IL-12, IL-23, IFN-y y TNF- $\alpha$ cuando el microorganismo estimulante fue la cepa ATCC ${ }^{\circledR} 43718^{\text {TM }}$ (serotipo b). Conclusión: El serotipo b de $A$. actinomycetemcomitans posee un mayor potencial inmuno-estimulador de células dendríticas comparado con los otros serotipos bacterianos y potencialmente contribuiría a inducir un patrón de respuesta inmune tipo Th1 y/o Th17 durante las periodontitis.
\end{abstract}

Rev. Clin. Periodoncia Implantol. Rehabil. Oral Vol. 6(2); 57-62, 2013.

Palabras clave: Células dendríticas, citoquinas, Aggregatibacter actinomycetemcomitans.

\begin{abstract}
Objective: A. actinomycetemcomitans expresses a number of virulence factors that contribute to direct tissue damage and, based on the antigenicity of LPS O-polysaccharide, distinct serotypes have been described. The aim of this study was to determine the pattern of cytokine expression and secretion on dendritic cells stimulated with $A$. actinomycetemcomitans serotypes $\mathrm{a}, \mathrm{b}$ and $\mathrm{c}$. Methods: Using different multiplicity of infections of the serotypes $\mathrm{a}, \mathrm{b}$, and $\mathrm{c}$ of $A$. actinomycetemcomitans, the mRNA expression and secretion levels for cytokines IL-1 $\beta$, IL-5, IL-6, IL-10, IL-12, IL-23, TNF- $\alpha$, and IFN- $\gamma$ were determined in stimulated dendritic cells using PCR and ELISA. Results: A dose-dependent increase in the secretion levels for IL-1 $\beta$, IL-5, IL-6, IL-10, IL-12, IL-23, TNF- $\alpha$, and IFN- $y$ was elicited on dendritic cells following stimulation with each of the serotypes of $A$. actinomycetemcomitans. In addition, $A$. actinomycetemcomitans serotype b (ATCC ${ }^{\circledR} 43718^{\mathrm{TM}}$ ) induced higher levels of IL-1 $\beta$, IL-6, IL-12, IL-23, IFN- $\gamma$ y TNF- $\alpha$ compared with the other strains. Conclusion: These data demonstrate that the distinct $A$. actinomycetemcomitans LPS O-polysaccharide serotypes induce both quantitative and qualitative differences in the dendritic cell response. Furthermore, the observed dendritic cell response to A. actinomycetemcomitans b serotype was characteristic of a Th1 and Th17 pattern of cytokine expression.
\end{abstract}

Rev. Clin. Periodoncia Implantol. Rehabil. Oral Vol. 6(2); 57-62, 2013.

Key words: Dendritic cells, citokines, Agregagibacter actinomycetemcomitans.

\section{INTRODUCCIÓN}

Las periodontitis son un conjunto de enfermedades de naturaleza inflamatoria y etiología infecciosa, cuya causa principal son las bacterias que residen en el biofilm patogénico subgingival ${ }^{(1,2)}$. Aunque las bacterias pueden causar dañar a los tejidos, es la respuesta inmune del hospedero la principal causa de la destrucción del aparato de soporte periodontal(3,4). Estas interacciones (bacteria-hospedero) inducen la síntesis de citoquinas y quimioquinas, las que pueden determinar la destrucción del tejido conectivo y óseo característico de las periodontitis $y$, eventualmente, pueden llevar a la pérdida de los dientes ${ }^{(5,8)}$.

En las periodontitis, las células dendríticas reconocen las bacterias patógenas del biofilm subgingival, las fagocitan y destruyen, y luego procesan y conjugan sus antígenos a moléculas del complejo mayor de histocompatibilidad (MHC) para presentarlos a los linfocitos $\mathrm{TCD}^{+}$y así, iniciar la respuesta inmune adaptativa(9-12). Las células dendríticas maduras secretan interleuquina (IL)-1ß, IL-5, IL-6, IL-10, IL-12, IL-23, interferon (IFN)- $\gamma$, factor de necrosis tumoral (TNF)- $\alpha$ y TNF- $\beta^{(13)}$, las que durante la presentación antigénica cumplen un rol fundamental en la activación y diferenciación de los linfocitos TCD4 ${ }^{+}$ a alguno de los fenotipos efectores: T-helper (Th)-1, Th2, Th17 o T reguladores (Treg) inducidos ${ }^{(14)}$. Los linfocitos cumplen un rol central en la inmunidad durante la infección periodontal y sus distintos fenotipos efectores determinan las características clínicas de la enfermedad $(12,15,16)$.

El biofilm patogénico subgingival, constituido principalmente por bacterias anaerobias Gram-negativo, es el factor etiológico responsable del inicio y progresión de las periodontitis ${ }^{(2,17-19)}$. Las bacterias periodonto-patógenas que componen este biofilm pueden causar daño directo a los tejidos periodontales; sin embargo, es la respuesta inmuno-inflamatoria inducida en el hospedero ante ellas la principal responsable de la destrucción de los tejidos de soporte de los dientes: ligamento periodontal, cemento radicular y hueso alveolar ${ }^{(5)}$.

1. Laboratorio de Biología Periodontal. Departamento de Odontología Conservadora. Facultad de Odontología, Universidad de Chile. Chile.

Correspondencia autor: Jaime Díaz. zaidemiaj@gmail.com / Rolando Vernal. rvernal@uchile.cl. Trabajo recibido el 06/09/2012. Aprobado para su publicación el 06/03/2013. 
Fusobacterium nucleatum, Porphyromonas gingivalis y Aggregatibacter actinomycetemcomitans son bacterias patógenas que han sido ampliamente asociadas a la etiología de las periodontitis ${ }^{(20)}$. Choi y cols. observaron una modulación de la respuesta inmune inducida por $P$. gingivalis ante la exposición previa del hospedero a F. nucleatum, simulando la infección bacteriana secuencial observada durante la periodontitis, evidenciando un cambio a una respuesta Th2 en vez de la respuesta inmune Th1 original(21). Además, Kopitar y cols. demostraron que el tipo de respuesta Th inducido depende del tipo de bacteria que estimula las células dendríticas, al diferenciarse un fenotipo linfocitario Th distinto ante extractos antigénicos preparados de Bacteroides fragilis, Streptococcus mitis y Propionibacterium acnes, en particular una respuesta tipo Th1, Th2 y Treg, respectivamente ${ }^{(22)}$. Estos datos sugieren que la respuesta inmune inducida depende del tipo de bacteria con la cual las células dendríticas son estimuladas.

Recientemente se ha demostrado que las distintas cepas bacterianas de una misma especie de microorganismo también se podrían asociar a la inducción de una variable respuesta inmune. En efecto, aunque la cepa $P$. gingivalis 381 , caracterizada por la presencia de fimbria, mostró un efecto inductor potente de la secreción de IL-12(23), al estimular las células dendríticas con la cepa $P$. gingivalis O55:B5 se detectó una débil respuesta inmuno-estimuladora de la secreción de IL-6, IL-8, IL-10, IL-12 y RANTES ${ }^{(24)}$. Confirmando estos datos, Jotwani y Cutler demostraron que la cepa $P$. gingivalis 381 con fimbria es más eficaz que la cepa mutante $P$. gingivalis DPG3 carente de fimbria en invadir y penetrar células dendríticas, inducir la expresión de HLA-DR, CD83, CD80 y CD86 y la secreción de IL-6, IL-10, IL-12 y TNF- $\alpha$ en células dendríticas, y estimular la proliferación y secreción de IFN-y en linfocitos $T^{(23)}$.

Sobre la base de la antigenicidad de la cápsula extracelular de $P$. gingivalis, se han descrito 6 serotipos capsulares $(\mathrm{K})$ diferentes: $\mathrm{K} 1-\mathrm{K} 6^{(25,26)}$. Al analizar la respuesta de las células dendríticas humanas ante estos distintos serotipos $\mathrm{K}$ de $P$. gingivalis se demostró que existen diferencias cuantitativas en la síntesis de citoquinas; en particular, los serotipos $\mathrm{K} 1$ y K2 mostraron un mayor potencial inmuno-estimulador comparado con los otros serotipos bacterianos ${ }^{(25)}$. La variable inmunogenicidad de los distintos serotipos $\mathrm{K}$ de $P$. gingivalis también ha sido demostrada en linfocitos TCD4 ${ }^{+}$, en los que indujeron una respuesta Th distinta. En efecto, un fenotipo tanto Th1 como Th17 se indujo en presencia de los serotipos K1 y K2, un fenotipo Th2 en presencia de los serotipos K3, K4 y K5 y un fenotipo Treg ante la estimulación con la cepa $\mathrm{K}^{-}$carente de cápsula extracelular ${ }^{(27,28)}$.

Sobre la base de la antigenicidad del polisacárido-O componente del lipopolisacárido (LPS) bacteriano, en A. actinomycetemcomitans se han identificado seis serotipos diferentes: a-f(29-31). Los serotipos de $A$. actinomycetemcomitans más frecuentemente detectados en la cavidad oral en humanos son $\mathrm{a}$, b y c, siendo el serotipo $\mathrm{b}$ el detectado con mayor frecuencia en pacientes afectados de periodontitis agresiva ${ }^{(32)}$. En este contexto, la mayor frecuencia de detección del serotipo b de A. actinomycetemcomitans en pacientes con periodontitis agresiva sugiere un mayor potencial patogénico y este podría asociarse a una mayor inmunogenicidad y virulencia ${ }^{(33)}$. En efecto, el serotipo b evade más eficazmente la fagocitosis por macrófagos ${ }^{(34)}$, inhibe con mayor intensidad la proliferación de macrófagos y neutrófilos ${ }^{(35)}$, produce mayores niveles de microvesículas citotóxicas ${ }^{(36)} \mathrm{e}$ induce un incremento en la secreción de $\lg G$, IgM e IgA por linfocitos B activados ${ }^{(29)}$. En efecto, el LPS es importante en $A$. actinomycetemcomitans dado que induce varibailidad en la respuesta inmune desplegada por el hospedero, a diferencia de los demás factores de virulencia (leucotoxina, toxina distensora citoletal, entre otros). Los distintos serotipos bacterianos podrían asociarse a un distinto potencial virulento, a una variable patogenicidad y a una asociación diferente en los estados de salud y enfermedad periodontal(20). Sin embargo, el rol de los distintos serotipos de $A$. actinomycetemcomitans aún no se ha definido en las células dendríticas.

La activación de la respuesta inmune involucra una serie de acontecimientos celulares y moleculares altamente coordinados cuyo rol fundamental es la defensa frente a la infección ${ }^{(38)}$ y las células dendríticas son determinantes esenciales del tipo de respuesta inmune desarrollada ante el biofilm ${ }^{(4,39)}$. En este estudio, se analizaron las diferencias en la síntesis de citoquinas producidas por células dendríticas humanas al ser estimuladas con los serotipos más frecuentemente detectados de $A$. actinomycetemcomitans: a, b y c.

\section{MATERIALES Y MÉTODOS}

\section{Cultivo y Recuento de Aggregatibacter actinomycetemcomitans}

Las cepas de $A$. actinomycetemcomitans ATCC $^{\circledR} 43717^{\text {TM }}$ (serotipo a), ATCC $^{\circledR} 43718^{\mathrm{TM}}$ (serotipo b) y ATCC $^{\circledR} 43719^{\mathrm{TM}}$ (serotipo c) se sembraron en medio de cultivo agar infusión cerebro-corazón (Oxoid Ltd., Hampshire, England) y fueron incubadas a $37^{\circ} \mathrm{C}$ en condiciones de capnofilia $\left(\mathrm{O}_{2} 8 \%\right.$ y CO, $\left.12 \%\right)$ usando generadores CampyGen ${ }^{\mathrm{TM}}$ (Oxoid Ltd.).

Para obtener número similar de microorganismos de los distintos serotipos de $A$. actinomycetemcomitans para la estimulación celular, cada una de las cepas bacterianas se inoculó en $5 \mathrm{~mL}$ de caldo de cultivo infusión cerebro-corazón (Oxoid Ltd.) hasta alcanzar un nivel McFarland de 5 (bioMérieux ${ }^{\circledR}$ SA, Marcy l'Etoile, France). Diluciones seriadas $\log _{10}$ de -1 a -9 fueron realizadas con amortiguador fosfato salino (PBS) estéril y $100 \mu \mathrm{L}$ de cada dilución fueron utilizados para la estimulación celular. El número de microorganismos de cada punto de estimulación celular fue establecido sembrando $100 \mu \mathrm{L}$ de las diluciones $-4,-5$ y - 6 en medio de cultivo agar infusión cerebro-corazón incubadas en condiciones de capnofilia. Luego de 2 días, el número de UFC/ $\mathrm{mL}$ de cada serotipo bacteriano fue establecido mediante recuento manual utilizando una lupa estereoscópica (STEMI 2000-C, Carl Zeiss Microscopy GMBH, Germany).

\section{Selección de los Sujetos}

Muestras de sangre periférica se obtuvieron de 8 sujetos donantes sanos (sin periodontitis $u$ otra enfermedad) voluntarios del banco de sangre del Hospital Dr. Luis Calvo Mackenna. A partir de las leuco-reducciones obtenidas durante el proceso de plaquetoféresis se purificaron los monocitos y linfocitos $\mathrm{TCD}^{+}$circulantes periféricos tal como se describe en detalle más adelante. El protocolo de investigación se explicó a todos los participantes del estudio, quienes firmaron un consentimiento informado aprobado por el Comité Ético-Científico de la Facultad de Odontología de la Universidad de Chile.

\section{Purificación de Monocitos}

A partir de las muestras de sangre obtenidas, se aislaron las células mononucleares (PBMCs) usando una gradiente de Ficoll ${ }^{\circledR}$ de acuerdo a las instrucciones del fabricante (Ficoll-Paque Plus, GE Healthcare, Uppsala, Sweden). Los monocitos se purificaron desde las PBMCs mediante separación inmuno-magnética (MACS; Miltenyi Biotec, Bergich Gladbach, Germany). Brevemente, las PBMCs se lavaron 4 veces en PBS EDTA 0,5 mM y luego se incubaron con un anticuerpo monoclonal (mAc) de ratón anti-CD14 humano conjugado con partículas inmuno-magnéticas durante $15 \mathrm{~min}$ a $4^{\circ} \mathrm{C}$. Posteriormente, las células se lavaron y se resuspendieron en $500 \mu \mathrm{L}$ de PBS frío y los monocitos se purificaron usando columnas de separación MS montadas en un campo electro-magnético. La fracción de células $\mathrm{CD}_{14}{ }^{+}$separadas corresponden a los monocitos, los cuales se contabilizaron usando una cámara de Neubauer y su fenotipo se confirmó usando anticuerpos específicos mediante citometría de flujo.

\section{Diferenciación y Estimulación de las Células Dendríticas}

Los monocitos purificados se sometieron inmediatamente a un protocolo estandarizado de diferenciación a células dendríticas. Los monocitos $\mathrm{CD}_{14}{ }^{+}$se cultivaron a una concentración de $1 \times 10^{6}$ células $/ \mathrm{mL}$ en $3 \mathrm{~mL}$ de medio de cultivo RPMI-1640, suplementado con $10 \%$ de suero bovino fetal (Gibco Invitrogen Corp., Grand Island, NY, USA) y $20 \mathrm{ng} / \mathrm{mL}$ de los factores estimulantes de colonias de granulocitos-macrófagos (GMCSF) e IL-4 (R\&D, Minneapolis, MN USA), durante 6 días. Las células dendríticas diferenciadas se estimularon con los distintos serotipos de $A$. actinomycetemcomitans a una multiplicidad infectante incremental (MOI) de $10^{-1}-10^{-2}$ (razón bacterias/células dendríticas) durante 2 días. Como control positivo, células dendríticas se estimularon con $10 \mathrm{ng} / \mathrm{mL}$ del LPS de Escherichia coli cepa 0111:B4 (Fluka, Sigma-Aldrich Chemie, Buchs, Switzerland). Células dendríticas no estimuladas se utilizaron como control negativo. La diferenciación y activación de las células dendríticas se evaluó con anticuerpos específicos mediante citometría de flujo. 


\section{Análisis de Fenotipo Celular}

La purificación de los monocitos, su diferenciación a células dendríticas y su posterior activación en presencia de los distintos serotipos de $A$. actinomycetemcomitans se cuantificó mediante citometría de flujo usando los siguientes mAc de ratón anti-humano conjugados con fluorocromos PE, FITC o PE/Cy5: anti-CD14 (monocitos), CD1a (células dendríticas inmaduras), y CD83 (células dendríticas maduras) (BD Biosciences Pharmigen, San José, CA, USA) y utilizando un equipo FACScan de citometría (Becton Dickinson, Franklin Lakes, NJ, USA).

\section{Expresión de las Moléculas Co-estimuladoras CD80 y CD86}

Con el objetivo de comparar los niveles de activación de las células dendríticas ante los distintos serotipos de $A$. actinomycetemcomitans, la expresión de las moléculas co-estimuladoras de la presentación antigénica CD80 y CD86 se evaluó mediante citometría de flujo usando $\mathrm{mAc}$ de ratón anti-humano conjugados con fluorocromos PE y FITC, respectivamente (BD Biosciences Pharmigen).

\section{Niveles de Expresión de mRNA de Citoquinas Analizada Mediante RT-PCR}

Luego de la estimulación celular, se procedió a la separación de las células dendríticas y el sobrenadante del cultivo celular mediante centrifugación a $5.000 \mathrm{xg}$ durante $10 \mathrm{~min}$

Las células dendríticas se lavaron dos veces en PBS y se lisaron en $400 \mu \mathrm{L}$ de solución de lisis Igepal $^{\circledR}$ CA-630 0.5\% (SigmaAldrich, Saint Louis, MO, USA), $50 \mathrm{mM}$ Tris- $\mathrm{HCl} \mathrm{pH} 8,100 \mathrm{mM} \mathrm{NaCl}$, $5 \mathrm{mM} \mathrm{MgCl}$ y $10 \mathrm{mM}$ de vanadyl-ribonucleosido (Gibco Invitrogen, Carlsbad, CA, USA). Luego de una centrifugación a $14.000 \mathrm{xg}$ durante $10 \mathrm{seg}$, el sobrenadante fue digerido con $25 \mu \mathrm{g} / \mathrm{mL}$ de proteinasa $\mathrm{K}$ (Roche Ltd, Basel, Switzerland), sulfato de sodio dodecil $1 \%$ y $15 \mathrm{mM}$ de EDTA (Fluka Sigma-Aldrich, St. Louis, MO, USA) durante 30 min a $37^{\circ} \mathrm{C}$. El RNA citoplasmático total fue extraído con $400 \mu \mathrm{L}$ de solución fenol:cloroformo:isoamylalcohol 25:24:1 (Fluka Sigma-Aldrich, St. Louis, $\mathrm{MO}$, USA), precipitado a $-20^{\circ} \mathrm{C}$ con $0,3 \mathrm{M}$ de acetato de sodio, $1 \mathrm{~mL}$ de etanol $100 \%$ y $20 \mu \mathrm{g} / \mu \mathrm{L}$ de glicógeno (Roche Ltd, Basel, Switzerland), recuperado por centrifugación a 14.000 xg durante 30 min y resuspendido en $30 \mu \mathrm{L}$ de agua libre de RNAsas. El RNA obtenido se evaluó utilizando un bioanalizador (Agilent 2100B; Agilent Technologies, Palo Alto, CA, USA) y se cuantificó usando un espectrofotómetro (Bio-Tek, Winooski, $\mathrm{VT}$, USA), para almacenarlo a $-80^{\circ} \mathrm{C}$ a una concentración final de $1 \mu \mathrm{g} /$ $\mu \mathrm{L}$. A partir de $2 \mu \mathrm{g}$ de RNA se procedió a la síntesis de la primera cadena de cDNA mediante transcripción reversa, utilizando el kit SuperScript ${ }^{\circledR}$ III First-Strand Synthesis SuperMix de acuerdo a las instrucciones del fabricante (Invitrogen, Grand Island, NY, USA).

La expresión de los mRNA de las citoquinas IL-1 $\beta$, IL-5, IL6, IL-10, IL-12, IL-23, IFN- $\gamma$ y TNF- $\alpha$ se analizó a partir de $50 \mathrm{ng} / \mu \mathrm{L}$ de cDNA mediante RT-PCR, utilizando partidores específicos (Tabla 1) y un kit GoTaq Green Master ${ }^{\circledR}$ (Promega Corp, Madison, WI, USA), siguiendo el siguiente protocolo de amplificación: $95^{\circ} \mathrm{C}$ durante 3 minutos, 40 ciclos de $95^{\circ} \mathrm{C}$ durante 30 segundos y $76^{\circ} \mathrm{C}$ durante 30 segundos y una incubación final a $74^{\circ} \mathrm{C}$ durante 5 minutos. La amplificación se evaluó mediante electroforesis en gel agarosa $2 \%$ revelado con GelRed ${ }^{\mathrm{TM}}$ (Biotium, CA, USA). Como control de amplificación se analizaron los niveles de expresión de la subunidad de RNA ribosomal $18 \mathrm{~S}$.

\section{Secreción de Citoquinas Analizada Mediante ELISA}

A partir de 100 ó $200 \mu \mathrm{L}$ del sobrenadante de cultivo celular se cuantificó la secreción de las citoquinas IL-1 $\beta$, IL-5, IL-6, IL-10, IL12, IL-23, IFN- $y$ y TNF- $\alpha$ mediante ELISA siguiendo las instrucciones del fabricante (R\&D, Minneapolis, USA) y evaluando la absorbancia a 460 nm y $560 \mathrm{~nm}$ usando un espectrofotómetro de placas (Bio-Tek, Winooski, VT, USA).

\section{Análisis Estadístico}

Los datos de citometría de flujo se representaron como histogramas usando el software WinMDi 2.9 (The Scripps Research Inst., La Jolla, CA, USA) y se expresaron como porcentaje promedio \pm desviación estándar de células positivas. Los niveles de citoquinas evaluados mediante ELISA se expresaron como concentración promedio \pm desviación estándar. Los datos se analizaron estadísticamente usando el software SPSS v.15.0 (Lead Technologies Inc., Charlotte, NC, USA). La normalidad de la distribución de los datos se determinó usando la prueba de Shapiro-Wilk. Las diferencias en la expresión de los marcadores de superficie CD80, CD83 y CD86 se analizaron mediante la prueba $x^{2}$ y los niveles de las citoquinas se analizaron utilizando la pruebas ANOVA y de Tukey o las pruebas de Kruskal-Wallis y de Dunn. Las diferencias se consideraron estadísticamente significativas cuando $p$-value $<0.05$.

Tabla 1. Partidores utilizados para la amplificación de los mRNA de las citoquinas mediante RT-PCR. La expresión de la subunidad 18S del RNA ribosomal (18S rRNA) se utilizó como control de amplificación.

\begin{tabular}{|c|c|c|}
\hline Citoquinas & Partidor Forward & Partidor Reverse \\
\hline IL-1 $\beta$ & ctgtcctgcgtgttgaaaga & ttgggtaatttttgggatctaca \\
\hline IL-5 & ctctgaggattcctgttcctgt & cagtacccccttgcacagtt \\
\hline IL-6 & gcccagctatgaactccttct & gaaggcagcaggcaacac \\
\hline IL-10 & tgggggagaacctgaagac & ccttgctcttgtttcacagg \\
\hline IL-12 & cactcccaaaacctgctgag & tctcttcagaagtgcaagggta \\
\hline IL-23 & agcttcatgcctccctactg & ctgctgagtctcccagtggt \\
\hline IFN- $\gamma$ & ggcatttgaagaattggaaag & tttggatgctctggtcatctt \\
\hline TNF- $\alpha$ & cagcctcttctccttcctgat & gccagagggctgattagaga \\
\hline $18 \mathrm{~S}$ rRNA & ctcaacacgggaaacctcac & cgctccaccaactaagaacg \\
\hline
\end{tabular}

\section{RESULTADOS}

A partir de las PBMCs aisladas de sangre periférica, se obtuvo una población de monocitos de alta pureza, demostrado por los elevados niveles de expresión del marcador específico CD14 (>97\%) (Figura 1). Un alto porcentaje de ellos ( $>98 \%$ ) se diferenció a células dendríticas ante la estimulación con GM-CSF e IL-4, tal como se demuestra por la pérdida del marcador de monocitos CD14 y el incremento en los niveles de expresión de CD1a (Figura 1).

La Figura 2 muestra los niveles de activación de las células dendríticas ante los distintos serotipos de $A$. actinomycetemcomitans. Tal como se demuestra por la elevada expresión del marcador de células dendríticas maduras CD83 y de las moléculas co-estimuladoras CD80 y CD86, los niveles de activación de las células dendríticas fueron altos (>95\%), sin detectarse diferencias entre los distintos serotipos de A. actinomycetemcomitans (CD83 $p=0.938$, CD80 $p=0.532$ y CD86 $\mathrm{p}=0.933$ ).

La expresión de los mRNA de las citoquinas IL-1 $1 \beta$, IL-5, IL6, IL-10, IL-12, IL-23, IFN- $\gamma$ y TNF- $\alpha$ en células dendríticas estimuladas con los distintos serotipos de $A$. actinomycetemcomitans $\left(\mathrm{MOI}=10^{-2}\right)$ se muestran en la Figura 3. La expresión de citoquinas fue característica de un patrón de respuesta tipo Th1 y Th17 y fue concordante con los niveles secretados a nivel de proteínas. En efecto, elevados niveles de IL-1 $\beta$, IL-6, IL-12, IL-23, IFN- $y$ y TNF- $\alpha$ fueron detectados, con un efecto dosisrespuesta dependiente de la $\mathrm{MOI}$ de microorganismos estimulantes (Figura 4). Los niveles de secreción de IL-5 e IL-10 fueron marginales, no observándose producción dependiente de la dosis.

Mayores niveles de IL-1 $\beta$, IL-12, IL-23, IFN- $\gamma$ y TNF- $\alpha$ fueron secretados por las células dendríticas estimuladas con el serotipo b de $A$. actinomycetemcomitans en comparación a los otros serotipos bacterianos (Figura 4). Estas diferencias fueron detectadas a $\mathrm{MOI}=10^{-2}$ para las citoquinas tipo Th1 IL-1 $\beta$ ( $p=0.048), \mathrm{IL}-12(p=0.042)$, IFN- $\gamma(p<0.0001)$ y TNF- $\alpha(p<0.001)$ y para la citoquina tipo Th17 IL-23 $(p=0.015)$, y a $\mathrm{MOI}=10^{-1}$ para las citoquinas tipo Th1 IFN- $\gamma(p=0.003)$ y TNF- $\alpha(p=0.002)$ y la citoquina tipo Th17 IL-23 $(p<0.001)$. Para las citoquinas tipo Th2 IL-5 y tipo Treg IL-10 no se detectaron diferencias entre los distintos serotipos de $A$. actinomycetemcomitans. Además, no se observaron diferencias de secreción de ninguna citoquina entre los serotipos a y c de A. actinomycetemcomitans. 


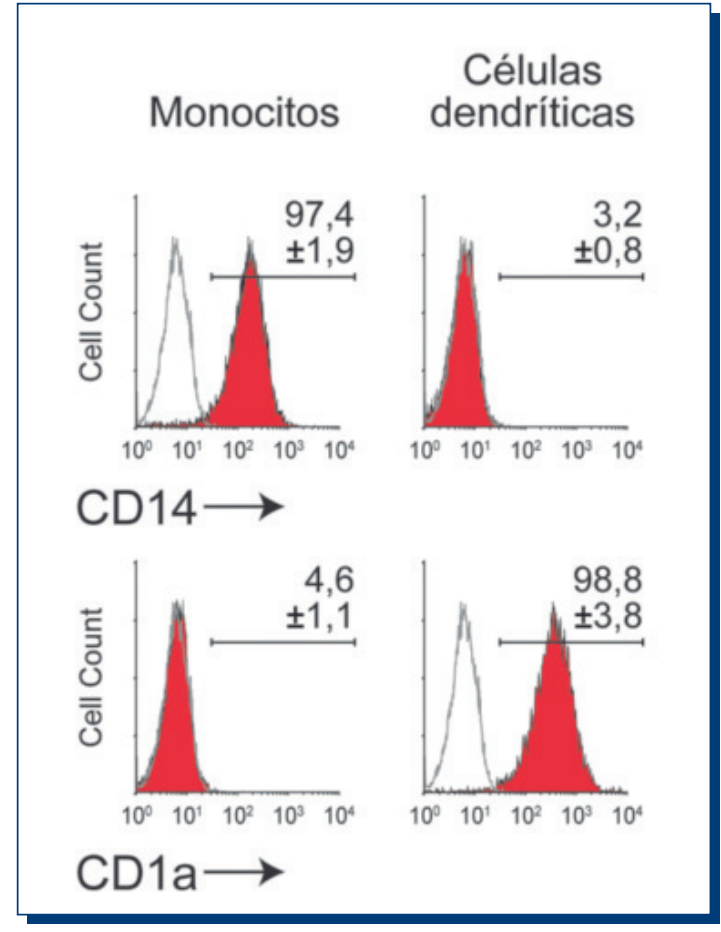

Figura 1. Purificación de monocitos y diferenciación de células dendríticas. Análisis mediante citometría de flujo de los niveles de purificación de monocitos (células $\mathrm{CD} 14^{+}$) a partir de las PBMCs y de su diferenciación a células dendríticas (células $\mathrm{CD}_{1} \mathrm{a}^{+}$) en presencia de GM-CSF e IL-4. Los histogramas mostrados son un experimento representativo y los valores de cada histograma representan el porcentaje (\%) promedio \pm desviación estándar de células positivas de 4 experimentos independientes.

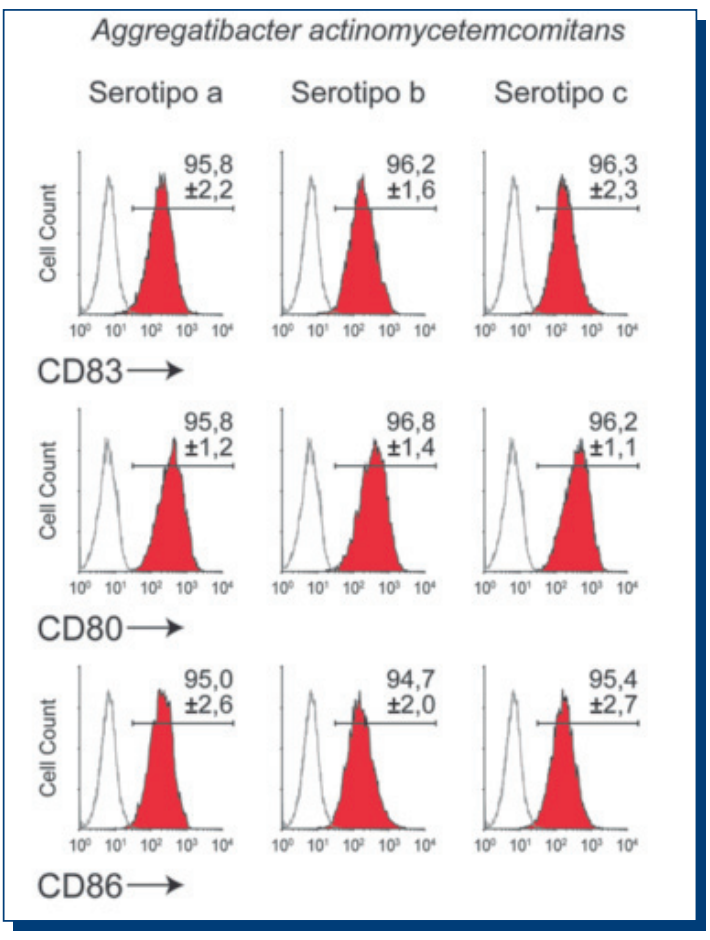

Figura 2. Activación de las células dendríticas ante los serotipos a, b y c de Aggregatibacter actinomycetemcomitans. Análisis mediante citometría de flujo de los niveles de expresión de CD83 (marcador de células dendríticas maduras), CD80 y CD86 (moléculas co-estimuladoras de la presentación antigénica) que evidencian la activación de las células dendríticas luego de la estimulación durante 48 horas con las cepas ATCC $^{\circledR} 43717^{\text {TM }}$ (serotipo a), ATCC ${ }^{\circledR} 43718^{\text {TM }}$ (serotipo b) y ATCC ${ }^{\circledR}$ $43719^{\mathrm{TM}}$ (serotipo c) de $A$. actinomycetemcomitans $\left(\mathrm{MOI}=10^{-2}\right.$ ). Los histogramas mostrados son un experimento representativo y los valores de cada histograma representan el porcentaje (\%) promedio \pm desviación estándar de células positivas de 4 experimentos independientes.

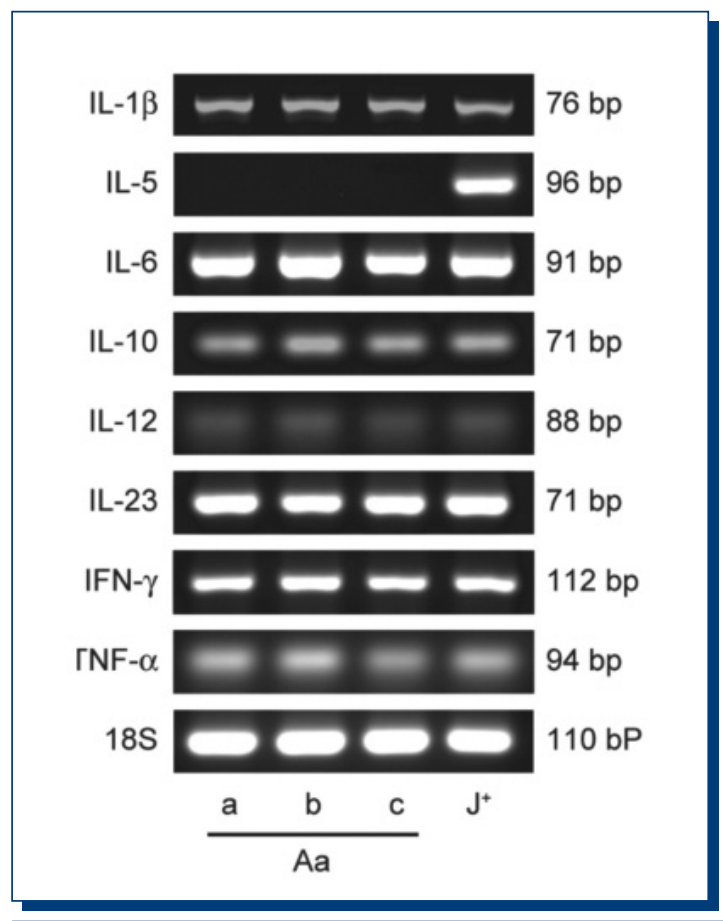

Figura 3. Expresión de citoquinas en células dendríticas estimuladas con los serotipos a, b y c de Aggregatibacter actinomycetemcomitans. La expresión de los mRNA de las citoquinas IL-1 $\beta$, IL-5, IL-6, IL-10, IL-12, IL-23, IFN-y y TNF- $\alpha$ fue determinada mediante RT-PCR en células dendríticas estimuladas durante 48 horas con las cepas ATCC $^{\circledR} 43717^{\text {TM }}$ (serotipo a), ATCC ${ }^{\circledR} 43718^{\text {TM }}$ (serotipo b) y ATCC ${ }^{\circledR} 43719^{\text {TM }}$ (serotipo c) de A. actinomycetemcomitans $\left(\mathrm{MOI}=10^{-2}\right)$. Como control positivo, la expresión de los mRNA de las citoquinas fue determinada en linfocitos T Jurkat E61.2 estimulados con los anticuerpos monoclonales anti-CD3 y CD28 $\left(\mathrm{J}^{+}\right)$. Como control de técnica, se analizaron los niveles de expresión de la subunidad rRNA 18S (18S). IFN: interferón, IL: interleuquina, MOI: multiplicidad infectante incremental (del inglés: multiplicity of infection) TNF: factor de necrosis tumoral.

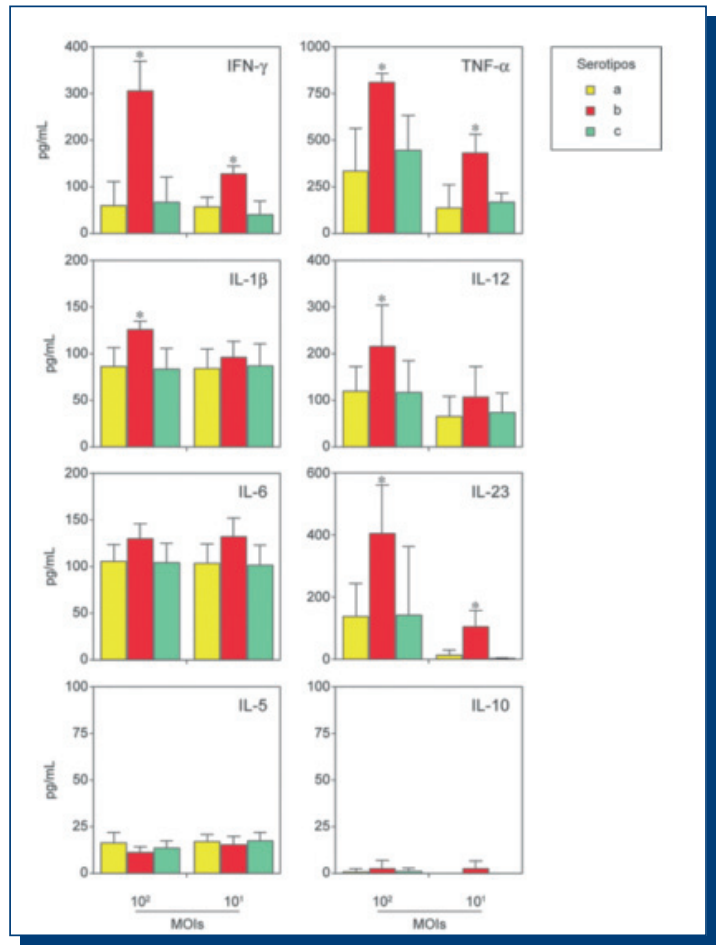

Figura 4. Secreción de citoquinas en células dendríticas estimuladas con los serotipos a, b y c de Aggregatibacter actinomycetemcomitans. La secreción de las citoquinas IL-1 $\beta$, IL-5, IL-6, IL-10, IL-12, IL-23, IFN-y y TNF- $\alpha$ fue cuantificada mediante ELISA en células dendríticas estimuladas durante 48 horas con las cepas ATCC ${ }^{\circledR} 43717^{\mathrm{TM}}$ (serotipo a), ATCC $^{\circledR} 43718^{\text {TM }}$ (serotipo b) y ATCC ${ }^{\circledR} 43719^{\text {TM }}$ (serotipo c) de A. actinomycetemcomitans (MOls $=10^{-1}$ y $10^{-2}$. Como control positivo, los niveles de secreción de citoquinas fueron cuantificados en células dendríticas estimuladas durante 48 horas con $10 \mathrm{ng} / \mathrm{mL}$ del LPS de Escherichia coli cepa 0111:B4 (LPS). La secreción de citoquinas se expresa como niveles promedio \pm desviación estándar de 8 experimentos independientes. IFN: interferón, IL: interleuquina, LPS: lipopolisacárido, MOI: multiplicidad infectante incremental (del inglés: multiplicity of infection), TNF: factor de necrosis tumoral. ${ }^{*} p<0.05$. 


\section{DISCUSIÓN}

En el presente estudio, se evaluó la respuesta de células dendríticas expuestas a distintos serotipos de $A$. actinomycetemcomitans analizando los niveles de expresión y secreción de citoquinas mediante PCR y ELISA, respectivamente. Los resultados obtenidos nos permiten establecer que el serotipo b de $A$. actinomycetemcomitans (cepa ATCC ${ }^{\circledR}$ $43718^{\mathrm{TM}}$ ) induce mayores niveles de IL-1 $\beta$, IL-6, IL-12, IL-23, IFN- $\gamma$ y TNF- $\alpha$, citoquinas del perfil Th1 y Th17, en comparación a los serotipos a y $c$.

IL-1 $\beta$ es una citoquina que media la inflamación y es conocida como un factor de activación de osteoclastos ${ }^{(40)}$, mientras que IL-6 participa en el proceso de diferenciación de linfocitos $B$ a células plasmáticas, la diferenciación selectiva de linfocitos Th17, la liberación de proteínas de fase aguda, la activación de la cascada del complemento y la inhibición de la diferenciación de linfocitos Treg Foxp3 ${ }^{+(41,42)}$. Además, IL-1 $\beta$, IL-6 y TNF- $\alpha$ tienen particular importancia en la maduración y migración de las células dendríticas, en la interacción de ellas con los Linfocitos TCD4 ${ }^{+}$durante la presentación antigénica y en la definición del estado clínico de la enfermedad ${ }^{(43)}$.

En forma similar a IL-1 $1 \beta$ e IL-6, un incremento en los niveles de IL-12 e IL-23 ha sido detectado en pacientes con periodontitis comparado con individuos sanos ${ }^{(44)}$ y estos se han asociado a la actividad de las células dendríticas ${ }^{(27,45)}$. Además, en conjunto con IL-6 y TGF- $\beta$, son capaces de inducir la diferenciación selectiva de linfocitos Th17(46).

Durante las periodontitis, IFN-y se ha detectado en elevados niveles de lesiones periodontales progresivas y/o severas ${ }^{(47)}$ y estos elevados niveles se han asociado a un incremento en la secreción de IL-1 $\beta$, IL-6 y TNF- $\alpha$ y a la pérdida de inserción conectiva y reabsorción ósea alveolar ${ }^{(48-50)}$.

La variabilidad en la secreción de citoquinas y la respuesta de los linfocitos $\mathrm{TCD}^{+}$durante las periodontitis podría explicarse por las diferencias de concentración y composición de patógenos o sus antígenos en los sitios periodontales infectados. En efecto, en el biofilm subgingival es posible encontrar diferentes concentraciones de bacterias periodonto-patógenas que dependen, por ejemplo, de las condiciones del micro-ambiente local ${ }^{(2)}$. Además, la concentración y composición del biofilm subgingival no sólo varía entre un individuo sano y un paciente con periodontitis, sino que también entre sitios sanos y lesiones periodontales en el mismo individuo(51), pudiendo relacionarse directamente con la presencia de un serotipo o bacteria en particular. Así, las variaciones en la frecuencia de detección de $A$. actinomycetemcomitans entre las distintas formas de periodontitis, la detección en sujetos sanos y la persistencia en el ambiente subgingival de sujetos sanos posterior al tratamiento periodontal podrían deberse a una distribución distinta de sus serotipos dependiente del estado clínico periodontal del sujeto ${ }^{(52)}$ y esta variable distribución estaría asociada a la potencial distinta virulencia y patogenicidad entre estos serotipos bacterianos.

Recientemente, se haestablecidoque $A$. actinomycetemcomitans posee una mayor capacidad inmunogénica en comparación a $P$. gingivalis ${ }^{(27)}$ y que entre los distintos serotipos de $A$. actinomycetemcomitans existe una variable asociación a la enfermedad ${ }^{(53)}$. En efecto, la mayor frecuencia de detección del serotipo $\mathrm{b}$ de $A$. actinomycetemcomitans en pacientes con periodontitis agresiva sugiere un mayor potencial patogénico del microorganismo, asociado a una mayor inmunogenicidad ${ }^{(33)}$.

En este estudio se evidenciaron los niveles de producción de citoquinas por las células dendríticas ante distintos serotipos de $A$ actinomycetemcomitans y se proyecta su potencial rol en la definición del tipo de linfocito Th predominante en las lesiones periodontales luego de la presentación antigénica. Así, sobre la base de los datos presentados, podemos especular que el serotipo $b$ de $A$. actinomycetemcomitans eventualmente induciría una diferenciación predominantemente de linfocitos Th1 y/o Th17.

Esta especulación es concordante con datos previos obtenidos por nuestro grupo de investigación. Cuando las células dendríticas fueron estimuladas con distintos serotipos capsulares $\mathrm{K}$ de $P$. gingivalis, se detectó que los serotipos $\mathrm{K} 1$ y K2 indujeron una mayor expresión de IL-1 $\beta$, IL-6, IL-12, IFN- $\gamma$, TNF- $\alpha$ y TNF- $\beta$, en comparación a los otros serotipos $^{(27)}$, y promovieron un patrón de respuesta tipo Th1 o Th17 en Linfocitos TCD4 ${ }^{+}$activados, con mayores niveles de secreción de IL$1 \beta$, IL-2, IL-6, IL-12, IL-17, IL-23, IFN-y, TNF- $\alpha$ y TNF- $\beta$, y expresión de T-bet y RORC2, genes master-switch que determinan la diferenciación selectiva de linfocitos Th1 y Th17, respectivamente.

En conclusión, el serotipo b de $A$. actinomycetemcomitans induce una respuesta inmune en células dendríticas de mayor intensidad en comparación a la inducida ante los serotipos a y c, con un predominio de un patrón de respuesta tipo Th1 y Th17 sobre la base de la secreción de citoquinas. Así, el serotipo b de A. actinomycetemcomitans potencialmente se asociaría a la inducción de una respuesta inmunoinflamatoria y osteo-destructiva durante las periodontitis y los serotipos a y c podrían asociarse a una ausencia de progresión de la enfermedad, a una remisión de la misma o a condiciones de salud periodontal. En este contexto, la variable virulencia entre los distintos serotipos de $A$. actinomycetemcomitans podría implicar un re-análisis de las estrategias actuales de identificación de patógenos periodontales y la determinación de su asociación a las periodontitis, recomendándose una re-definición que involucre la variabilidad serotípica definida en relación al factor de virulencia más importante del microorganismo estudiado, tal como es el LPS de $A$. actinomycetemcomitans.

\section{AGRADECIMIENTOS}

Este trabajo fue financiado por los Proyectos FIOUCh 09-8, de la Facultad de Odontología de la Universidad de Chile, y FONDECYT 11100298, de la Comisión Nacional de Investigación Científica y Tecnológica CONICYT del Gobierno de Chile.

\section{CONFLICTOS DE INTERÉS}

Los autores declaran no tener conflictos de interés.

\section{REFERENCIAS BIBLIOGRÁFICAS}

1. Armitage G. Development of a classification system for periodontal diseases and conditions. Ann Periodontol, 1999; 4(1): 1-110.

2. Socransky S, Haffajee A. Periodontal microbial ecology. Periodontol 2000, 2005; 38: $135-187$.

3. Teng YT. Immune cell involvement in periodontal bone loss. In: Henderson B, Curtis MA, Seymour RM, Donos N editors. Periodontal Medicine and Systems Biology, Wiley Blackwell; 2009: 407-426.

4. Gemmell E, Yamazaki K, Seymour G. The role of T cells in periodontal disease: Homeostasis and autoimmunity. Periodontol 2000, 2007; 43: 14-40.

5. Offenbacher S. Periodontal diseases: Pathogenesis. Ann Periodontol, 1996; 1 : 812-869.

6. Salvi G, Lang N. Host response modulation in the management of periodontal diseases. J Clin Periodontol, 2005; 32(Suppl 6): 108-129.

7. Salvi G, Kandylaki M, Troendle A, Persson G, Lang N. Experimental gingivitis in type I diabetics: A controlled clinical and microbiological study. J Clin Periodontol, 2005; 32: 310-316.

8. Listgarten M. Pathogenesis of periodontitis. J Clin Periodontol, 1986; 13(5): 418430
9. Kikkert R, Laine M, Aarden L, Winkelhoff AV. Activation of toll-like receptors 2 and 4 by gram-negative periodontal bacteria. Oral Microbiol Immunol, 2007; 22(3): 145-151.

10. Ukai T, Mori Y, Onoyama M, Hara Y. Immunohistological study of interferon-gand interleukin-4-bearing cells in human periodontitis gingiva. Arch Oral Bio, 2001; 46: 901-908.

11. Nussbaum G, Ben-Adi S, Genzler T, Sela M, Rosen G. Involvement of toll-like receptor 2 and 4 in the innate immune response to Treponema denticola and its outer sheath components. Infect Immun, 2009; 77: 3939-3947.

12. Garlet G. Destructive and protective roles of cytokines in periodontitis: A reappraisal from host defense and tissue destruction viewpoints. J Dent Res, 2010 89(12): 1349-1363.

13. Brightbill H, Libraty D, Krutzik S, Yang R, Belisle J, Bleharski J, Maitland M, Norgard M, Plevy S, Smale S et al. Host defense mechanism triggered by microbial lipoproteins throught toll-like receptors. Science, 1999; 285: 732-736.

14. Zhu J, Paul W. CD4 T cells: Fates, functions and faults. Blood, 2008; 112(5) 1557-1569. 
15. Steinman R, Hawiger D, Nussenzweig M. Tolerogenic dendritic cells. Annu Rev Immunol, 2003; 21: 685-711.

16. Gemmell E, Seymour G. Immunoregulatory control of Th1/Th2 cytokine profiles in periodontal disease. Periodontol 2000, 2004; 35: 21-41.

17. Socransky S, Haffajee A. Dental biofilms: Difficult therapeutic targets. Periodontol 2000, 2002; 28: 12-55.

18. Curtis M, Slaney J, Aduse-Opoku J. Critical pathways in microbial virulence. $J$ Clin Periodontol, 2005; 32(6): 28-38.

19. Slots J, Bragd L, Wikstrom M, Dahle G. The occurrence of Actinobacillus actinomycetemcomitans, Bacteroides gingivalis and Bacteroides intermedius in destructive periodontal disease in adults. J Clin Periodontol, 1986; 13: 570-577. 20. Díaz J, Yáñez J, Melgar S, Álvarez C, Rojas C, Vernal R. Virulencia y variabilidad de Porphyromonas gingivalis y Aggregatibacter actinomycetemcomitans y su asociación a la periodontitis. Rev Clín Per Imp Rehab Oral, 2012; 5(1): 40-45.

21. Choi J, Borrello M, Smith E, Cutler C, Sojar H, Zauderer M. Prior exposure of mice to Fusobacterium nucleatum modulates host response to Porphyromonas gingivalis. Oral Microbiol Immunol, 2001; 16: 338-344.

22. Kopitar A, Ahan Hren N, Ihan A. Commensal oral bacteria antigens prime human dendritic cells to induce Th1, Th2 or Treg differentiation. Oral Microbiol and Immunol, 2006; 21: 5.

23. Jowatni R, CW C. Fimbriated Porphyromonas gingivalis is more efficient than fimbria-deficient $P$. gingivalis in entering human dendritic cells in vitro and induces an inflammatory Th1 effector response. Infect Immun, 2004; 72(3): 1725-1732.

24. Kanaya S, Nemoto E, Ogawa T, Shimauchi H. Porphyromonas gingivalis lipopolysaccharides induce maturation of dendritic cells with CD14+CD16+ phenotype. Eur J Immunol, 2004; 34(5): 1451-1460.

25. Laine M, van WinkelhoffA. Virulence of six capsular serotypes of Porphyromonas gingivalis in a mouse model. Oral Microbiol Immunol, 1998; 13(5): 322-325.

26. Laine M, Appelmelk B, van Winkelhoff A. Novel polysaccharide capsular serotypes in Porphyromonas gingivalis. J Periodont Res, 1996; 31(4): 278-284.

27. Vernal R, Leon R, Silva A, van Winkelhoff A, Garcia-Sanz J, Sanz M. Differential cytokine expression by human dendritic cells in response to different Porphyromonas gingivalis capsular serotypes. J Clin Periodontol, 2009; 36(10): 823-829.

28. Vernal R, Diaz-Guerra E, Silva A, Sanz M, Garcia-Sanz J. Distinct Th responses triggered in human T lymphocytes by different Porphyromonas gingivalis capsular serotypes. J Clin Periodontol, 2012. En prensa.

29. King E, Tatum H. Actinobacillus actinomycetemcomitans and Haemophilus aphrophilus. J Infect Dis, 1962; 111: 85-94.

30. Kaplan J, Perry M, MacLean L, Furganga D, Wilson M, Fine D. Structural and genetic analyses of $O$ polysaccharide from Actinobacillus actinomycetemcomitans serotype f. Infect Immun, 2001; 69(9): 5375-5384.

31. Zambon J, Slots J, Genco R. Serology of Actinobacillus actinomycetemcomitans and serotype distribution in human periodontal disease. Infect Immun, 1983; 41(1): 19-27.

32. Cortelli J, Aquino D, Cortelli S, Roman-Torres C, Franco G, Gomez R, Batista L, Costa F. Aggregatibacter actinomycetemcomitans serotypes infections and periodontal conditions: A two-way assessment. Eur J Clin Microbiol Infect Dis, 2012; 31(7): 1311-1318.

33. Shimada T, Sugano N, Nishihara R, Suzuki K, Tanaka H, Ito K. Differential effects of five Aggregatibacter actinomycetemcomitans strains on gingival epithelial cells. Oral Microbiol Immunol, 2008; 23(6): 455-458.

34. Asikainen S, Lai C, Alaluusua S, Slots J. Distribution of Actinobacillus actinomycetemcomitans serotypes in periodontal health and disease. Oral Microbiol Immunol, 1991; 6(2): 115-118.

35. Suzuki N, Nakano Y, Yoshida Y, Ikeda D, Koga T. Identification of Actinobacillus actinomycetemcomitans serotypes by multiplex PCR. J Clin Microbiol, 2001; 39(5): 2002-2005.
36. Lai C, Listgarten M, Hammond B. Comparative ultrastructure of leukotoxic and non-leukotoxic strains of Actinobacillus actinomycetemcomitans. J Periodont Res, 1981; 16(4): 379-399.

37. Ebersole J, Sandoval M, Steffen M, Cappelli D. Serum antibody in Actinobacillus actinomycetemcomitans-infected patients with periodontal disease. Infect Immun, 1991; 59(5): 1795-1802.

38. Azuma M. Fundamental mechanisms of host immune responses to infection. $J$ Periodont Res, 2006; 41(5): 361-373.

39. Cutler C, Jowatni R. Antigen-presentation and the role of dendritic cells in periodontitis. Periodontol 2000, 2004; 35: 135-157.

40. Wilton J, Bampton J, Griffiths G, Curtis M, Life J, Johnson N, Powell J, Harrap G, Critchley P. Interleukin-1 beta (IL-1 beta) levels in gingival crevicular fluid from adults with previous evidence of destructive periodontitis. A cross sectional study. $J$ Clin Periodontol, 1992; 19(1): 53-57.

41. Revel M. Host defense against infections and inflammations: Role of the multifunctional IL6/IFN-beta cytokine. Experentia, 1989; 45(6): 549-558.

42. Korn T, Mitsdoerffer M, Croxford A, Awasthi A, Dardalhon V, Galileos G, Vollmar P, Stritesky G, Kaplan M, Waisman A et al. IL-6 controls Th17 immunity in vivo by inhibiting the conversion of conventional $T$ cells into Foxp3+ regulatory $T$ cells. PNAS, 2008; 105(47): 5 .

43. Gaffen S, Hajishengallis G. A new inflammatory cytokine on the block: Rethinking periodontal disease and the Th1/Th2 paradigm in the context of Th17 cells and IL-17. J Dent Res, 2008; 87(9): 817-826.

44. Johnson R, Serio F. Interleukin-18 concentrations and the pathogenesis of periodontal disease. J Periodontol, 2005; 76(5): 785-790.

45. Sasaki H, Suzuki N, Kent R, Kawashima N, Takeda J, Stashenko P. T cell response mediated by myeloid cell-derived IL-12 is responsible for Porphyromonas gingivalis-induced periodontitis in IL-10-deficient mice. J Immunol, 2008; 180(9): 6193-6198.

46. Vernal R, Garcia-Sanz J. Th17 and Treg cells, two new lymphocyte subpopulations with a key role in the immune response against infection. Infect Disord Drug Targets, 2008; 8(4): 207-220.

47. Dutzan N, Vernal R, Hernandez M, Dezerega A, Rivera O, Silva N, Aguillon J Puente J, Pozo P, Gamonal J. Levels of interferon-gamma and transcription factor T-bet in progressive periodontal lesions in patients with chronic periodontitis. $J$ Periodontol, 2009; 80(2): 290-296

48. Graves D, Fine D, Teng Y, Van Dyke T, Hajishengallis G. The use of rodent models to investigate host-bacteria interactions related to periodontal diseases. $J$ Clin Periodontol, 2008; 35(2): 89-105.

49. Graves D, Cochran D. The contribution of interleukin-1 and tumor necrosis factor to periodontal tissue destruction. J Periodontol, 2003; 74(3): 391-401.

50. Garlet G, Cardoso C, Campanelli A, Ferreira B, Avila-Campos M, Cunha F Silva J. The dual role of p55 tumour necrosis factor-alpha receptor in Actinobacillus actinomycetemcomitans-induced experimental periodontitis: Host protection and tissue destruction. Clin Exp Immunol, 2007; 147(1): 128-138.

51. Colombo A, Silva C, Haffajee A, Colombo A. Identification of oral bacteria associated with crevicular epithelial cells from chronic periodontitis lesions. J Med Microbiol, 2006; 55(5): 609-615.

52. Asikainen S, Chen C, Slots J. Actinobacillus actinomycetemcomitans genotypes in relation to serotypes and periodontal status. Oral Microbiol Immunol, 1995; 10(2): 65-68.

53. Henderson B, Ward JM, Ready D. Aggregatibacter (Actinobacillus) actinomycetemcomitans: A triple $A^{*}$ periodontopathogen? Periodontol 2000, 2010; 54(1): 78-105 